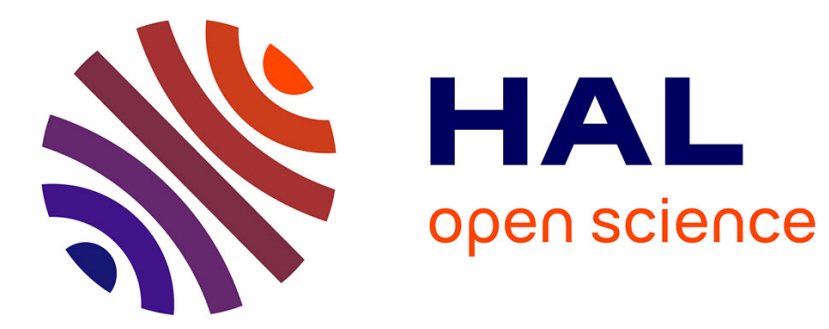

\title{
La vie privée, un bien commun ?
}

Paola Tubaro

\section{- To cite this version:}

Paola Tubaro. La vie privée, un bien commun ?. Regards croisés sur l'économie, 2019, 23 (2), pp.129137. 10.3917/rce.023.0129 . hal-02196333

\section{HAL Id: hal-02196333 https://hal.science/hal-02196333}

Submitted on 27 Jul 2019

HAL is a multi-disciplinary open access archive for the deposit and dissemination of scientific research documents, whether they are published or not. The documents may come from teaching and research institutions in France or abroad, or from public or private research centers.
L'archive ouverte pluridisciplinaire HAL, est destinée au dépôt et à la diffusion de documents scientifiques de niveau recherche, publiés ou non, émanant des établissements d'enseignement et de recherche français ou étrangers, des laboratoires publics ou privés. 


\section{La vie privée, un bien commun ?}

\section{Paola Tubaro, CNRS-LRI}

Résumé : La vie privée s'est transformés sous l'effet des technologies numériques et des modèles d'affaires des plateformes. L'ancienne notion de privacy comme pénétration dans l'espace intime d'une personne a laissé la place à une privacy «comme négociation », contextuelle et évolutive, dépendant de l'environnement social de chacun. Cette négociation doit être collective et associer les droits des usagers aux enjeux généraux de l'économie et du travail de la donnée.

Abstract: Digital technologies and platform business models have transformed privacy. The old notion of privacy as penetration into a person's intimate space has given way to privacy "as negotiation": contextual, evolving, and depending on each person's social environment. This negotiation must be collective and associate the rights of users with the general stakes of the data economy and data labor.

Avec l'essor du web participatif et des médias sociaux il y a déjà une dizaine d'années, la vie privée a pu paraître un concept révolu. Le déluge d'informations personnelles sur les profils en ligne des usagers et leur utilisation désinvolte par les fournisseurs de services ont fait croire à un glissement vers des valeurs de « publitude»(Jarvis, 2011). En 2013 Mark Zuckerberg, le fondateur de Facebook, disait que « That social norm is just something that has evolved over time ${ }^{1}$.

Fast forward en 2018 : avec le Règlement Général sur la Protection des Données (RGPD), les pays européens s'imposent de nouvelles mesures respectueuses de la vie privée. Le dernier rapport annuel de la Commission Nationale de 1'Informatique et des Liberté (CNIL), l'autorité française de protection des données personnelles, compte un nombre record de plaintes (8360 en 2017, contre 7703 en 2016), et « constate une préoccupation croissante des personnes quant à l'utilisation qui est faite de leurs données ». Que s'est-il donc passé ?

La réalité est que la «fin » annoncée de la vie privée n'a jamais eu lieu : le concept

\footnotetext{
${ }^{1}$ Interview avec M. Arrington, The Crunchies, Palace of Fine Arts Theater, San Francisco, 8 janvier 2013.
} 
s'est pourtant transformé sous l'effet des technologies numériques et des modèles d'affaires y afférant.

\section{D’une «privacy comme pénétration » à une «privacy comme négociation »}

Pour saisir ces changements, il convient de revenir sur les étapes principales de la construction du concept de vie privée, qui a connu de multiples mutations au cours de l'histoire (Ariès \& Duby, 1999). Sa signification moderne tire ses origines de la définition des juristes américains Samuel Warren et Louis Brandeis (1890) qui, ayant à l'esprit les célébrités poursuivies par des paparazzis, voyaient the right to privacy comme « le droit d'être laissé en paix ».

Cette notion de «privacy comme pénétration» était encore à la base de la loi informatique et libertés de 1978 en France, qui avait le mérite d'en étendre l'application à tout individu, connu du grand public ou non, dans la mesure où le développement pressenti des ordinateurs aurait pu l'exposer à des intrusions. Les réglementations de cette période (y compris un Règlement européen de 1995, qui suivit la législation française et homogénéisa les approches des pays membres en la matière) imposaient le consentement de l'individu à l'enregistrement, au traitement et à la sauvegarde de ses données, pour qu'une pénétration de son intimité ne soit pas considérée comme une violation.

Le consentement de l'individu n'est plus suffisant aujourd'hui, parce que les données sont connectées : elles incluent des informations sur les relations sociales, qui engagent des tiers (famille, amis, collègues, camarades de classe). C'est pour cultiver son réseau (on dirait en sociologie, pour accroître son « capital social ») que l'individu dévoile des aspects de sa vie personnelle en ligne: les photos de ses vacances, l'annonce de la fête d'anniversaire de son enfant. L'idée de se défendre contre des

pénétrations venant de l'extérieur se vide de sens : nos informations personnelles ne sont plus notre apanage exclusif mais se dispersent sur les profils Facebook, Twitter ou LinkedIn de nos amis. La vie privée est encastrée dans un tissu social, et « il n’y a rien de plus collectif qu'une donnée personnelle » (Casilli \& Tubaro, 2018).

La notion de privacy comme pénétration laisse alors la place à une notion de privacy « comme négociation » (Tubaro, Casilli \& Sarabi, 2014), sans noyau prédéfini 
d'informations à protéger, mais avec évaluation sélective et toujours renouvelée de quoi révéler à qui. Il s'agit d'un processus d'apprentissage où l'individu se dévoile peu à peu, s'ajustant progressivement aux réactions des autres et au contexte (Nissenbaum, 2009). Mettre en ligne les photos des vacances n'implique alors pas toujours renonciation complète à la vie privée : on peut faire des concessions sur des aspects (par exemple pour s'attirer la bienveillance de ses amis), sans reculer sur d'autres. Aussi, des informations considérées comme privées voire sensibles dans un contexte ne le sont pas dans un autre : on ne dévoile pas sa vie sexuelle sur Facebook mais on peut le faire sur un forum spécialisé - où par contre, on ne nomme surtout pas ses enfants.

Les technologies ont donc changé notre vision, auparavant monodirectionnelle et réservée à une élite, qui envisageait la vie privée comme un noyau d'information personnelle exposée au risque de pénétration depuis l'extérieur. Nous en faisons aujourd'hui une négociation, ouverte à tous, évolutive et contextuelle, dans une société numérisée et connectée. Au fond, les technologies ont étendu notre compréhension de la vie privée et augmenté (non diminué !) nos attentes.

\section{Négociation, inégalités et conflits}

Mais alors, pourquoi a-t-on pu croire en la fin de la vie privée ? Une raison est que les usagers ne sont pas égaux dans leur capacité à négocier. S'il est assez aisé de le faire avec les membres de son réseau social, il est plus difficile de maitriser la capture d'information par la plateforme même qui gère le service de réseautage en ligne, et l'éventuelle revente ou réutilisation de cette information. Les individus sont seuls face aux géants du web. Même parmi les millennials supposés bien connaître les technologies numériques, le statut socioéconomique et le niveau des compétences informatiques affectent fortement la capacité à ajuster les paramètres de confidentialité (Hargittai, 2010). Involontaires, ces erreurs peuvent résulter en une moindre protection de la vie privée.

Une autre raison est que la négociation peut être conflictuelle. Des groupes d'usagers de médias sociaux se sont souvent mobilisés à l'encontre de conditions perçues comme abusives : un exemple récent est la plainte de l'ONG Europe v. 
Facebook, mettant en cause les politiques de la plateforme en matière de protection de la vie privée. Malgré leur fréquence, ces initiatives n’ont pas entamé la puissance des grandes entreprises numériques.

\section{L’économie des données personnelles}

Si les plateformes entravent le processus de négociation, c'est à cause de la valeur économique des données personnelles. Mieux elles connaissent les usagers, plus elles peuvent segmenter finement le marché et en tirer profit, comme un annonceur qui proposerait des publicités ciblées susceptibles d'amener plus aisément à l'achat. La valeur des données s'accroît par leur structure en réseau : par exemple, le ciblage publicitaire gagne en efficacité lorsque l'usager se voit proposer un produit que l'un de ses amis, aux goûts très proches, a déjà apprécié. Inciter l'usager à se dévoiler de plus en plus tout en élargissant son réseau social et/ou en intensifiant les interactions avec ses membres, a été alors une stratégie de croissance des géants du web.

Antonio A. Casilli (2013) montre que des entreprises technologiques comme Google et Facebook ont tenté de se faire «entreprises de morale », au sens d'Howard Becker (1963), en s'efforçant de sensibiliser le public aux avantages présumés de la transparence en ligne, et de prôner un changement des règles. Les propos de Zuckerberg cités plus haut s'interprètent comme un discours d'accompagnement, fonctionnel à cet objectif.

Cette stratégie n'a finalement pas abouti et a été progressivement abandonnée, les entreprises se montrant actuellement plus attachées à la protection de la vie privée. Des pratiques douteuses n'ont pas pour autant disparu, et des scandales continuent d'éclater, comme le cas très médiatisé de la fuite de données imputée à Cambridge Analytica en 2017.

\section{Travail, production de données et vie privée}

Les modes de production des données, liés aux modèles d'affaires des plateformes, font peser de nouvelles menaces sur la vie privée.

Les données brutes produites par les usagers seraient inutilisables sans un travail 
important d'enrichissement, codage, étiquetage, mise en forme. Les photos des vacances en ligne peuvent, par exemple, être labélisées par des tags qui identifient les lieux, la saison, ou les commerces présents. Mieux ces photos sont annotées, mieux elles peuvent être exploitées économiquement, que ce soit pour vendre des espaces publicitaires à des annonceurs ou pour entraîner des intelligences artificielles censées proposer des voyages personnalisés. Pour ce faire, les entreprises ont recours à du micro-travail payé à la pièce. Par des plateformes comme Amazon Mechanical Turk, Clickworker ou Microworkers, elles peuvent solliciter l'apport de foules d'ouvriers du clic, souvent localisés dans des pays à faible coût de la main-d'œuvre, qui réaliseront des annotations pour quelques centimes.

Le micro-travail pose un double défi à la protection de la vie privée. D'une part, il se peut que des informations à caractère personnel soient confiées à des tiers, notamment à l'étranger, sans que la personne concernée en soit consciente. Outre les plateformes sociales, l'inquiétude concerne les objets connectés comme les assistants virtuels : les mots que les usagers croient avoir adressés à un robot sont parfois écoutés et transcrits par des humains, chargés de vérifier le bon fonctionnement des algorithmes de reconnaissance vocale, et de corriger d'éventuelles erreurs. En laissant dans l'ombre ces interventions, les entreprises dissimulent les limites des technologies et en soutiennent la vente.

D'autre part, dans la mesure où ces nouvelles formes de digital labor concernent des prestataires majoritairement précaires et peu rémunérés, d'anciens acquis en termes de conditions de travail et de répartition de son produit sont mis en cause. Comme le proposent Lionel Maurel et Laura Aufrère (2018), il faut alors associer les enjeux liés à la vie privée au droit du travail et à la protection sociale. Cela exige de repenser à nouveau la vie privée pour y voir un «bien commun », en sortant du cadre individualiste de la réglementation actuelle sur les données personnelles (y compris le RGPD), et des solutions consistant à autoriser la «vente » des données (Arrieta-Ibarra et al. 2018) dont les usagers seraient inégalement capables de bénéficier.

Voir la vie privée comme un bien commun revient à envisager des formes de négociation d'emblée collective. Il s'agit de prendre en compte l'encastrement de la vie privée dans le social (les relations de chacun) et l'économique (la chaîne de 
production de valeur des plateformes). De nouvelles solutions doivent être inventées au croisement de ces trois univers, pour que la défense des données personnelles s'allie à celle des droits des travailleurs de la donnée. La vie privée sera une richesse dont nous partagerons les fruits si nous arrivons à repenser plus globalement notre rapport aux technologies, aux systèmes de production, et au final au collectif.

\section{Bibliographie}

ARIES P., DuBy G. (1999), Histoire de la vie privée, Points, 1999.

ArrietA-IBARra I., GofF L., JimeneZ-Hernandez D., LANIER J., WeYL E.G. (2018). «Should we treat data as labor? Moving beyond «free » », AEA Papers and Proceedings, vol. 108, pp. 38-42.

BECKER H.S. (1963), Outsiders: Studies in the Sociology of Deviance, Free Press.

CASILli A.A. (2013), «Contre l'hypothèse de la « fin de la vie privée » », Revue française des sciences de l'information et de la communication, vol. 3. URL : http://journals.openedition.org/rfsic/630.

CAsilli A.A., Tubaro P. (2018), « Notre vie privée, un concept négociable », Le Monde, 24 janvier.

CNIL. (2018), Rapport annuel - CNIL en bref 2018. URL : https://www.cnil.fr/sites/default/files/atoms/files/cnil_en_bref_2018.pdf

HARGitTAI E. (2010), «Digital na(t)ives ? Variation in Internet skills and uses among members of the 'Net generation' », Sociological Inquiry, vol. 80, pp. 92-113.

JARVIS J. (2011), Public Parts: How Sharing in the Digital Age Improves the Way we Work and Live, Simon \& Schuster.

MAurel L., Aufrere L. 2018. «Pour une protection sociale des données personnelles », blog, 5 février. URL : https://scinfolex.com/2018/02/05/pour-uneprotection-sociale-des-donnees-personnelles/

Nissenbaum H. (2009), Privacy in Context. Technology, Policy, and the Integrity of Social Life, Stanford University Press.

Tubaro P., Casilli A.A., SArabi Y. (2014), Against the Hypothesis of the "End of Privacy”: An Agent-based Modelling Approach to Social Media. Springer. 
WARREN S., BRANDEIS L. (1890) «The right to privacy », Harvard Law Review, vol. 4, pp. 193-220. 